\title{
An accelerating algorithm for globally solving nonconvex quadratic programming
}

Li Ge ${ }^{1,2^{*}}$ id and Sanyang Liu

\section{"Correspondence:}

geli163@163.com

${ }^{1}$ School of Mathematics and

Statistics, Xidian University, Xi'an,

China

${ }^{2}$ School of Mathematical Sciences,

Henan Institute of Science and

Technology, Xinxiang, China

\begin{abstract}
To globally solve a nonconvex quadratic programming problem, this paper presents an accelerating linearizing algorithm based on the framework of the branch-and-bound method. By utilizing a new linear relaxation approach, the initial quadratic programming problem is reduced to a sequence of linear relaxation programming problems, which is used to obtain a lower bound of the optimal value of this problem. Then, by using the deleting operation of the investigated regions, we can improve the convergent speed of the proposed algorithm. The proposed algorithm is proved to be convergent, and some experiments are reported to show higher feasibility and efficiency of the proposed algorithm.
\end{abstract}

Keywords: Nonconvex quadratic programming; Global optimization; Linear relaxation approach; Deleting technique; Branch and bound

\section{Introduction}

In this paper, we consider the following nonconvex quadratic programming problem (NQP):

$$
(\mathrm{NQP}):\left\{\begin{array}{l}
\min f(y)=c^{T} y+y^{T} Q y \\
\text { s.t. } \quad y \in Y^{0}=\left\{y \in R^{n} \mid A y \leq b\right\}
\end{array}\right.
$$

where $Q=\left(q_{j k}\right)_{n \times n}$ is a real $n \times n$ symmetric matrix; $A=\left(a_{j k}\right)_{m \times n}$ is a real $m \times n$ matrix, and $c, y \in R^{n} ; b \in R^{m}$.

The (NQP) is an important class of global optimization problems, and it is not only because they have broad applications in the fields of statistics and design engineering, optimal control, economic equilibria, financial management, production planning, combinatorial optimization, and so on [1,2], but also because many other nonlinear problems can be transformed into this form [3-7], such as the following linear multiplicative programming (LMP) problem:

$$
(\mathrm{LMP}):\left\{\begin{array}{l}
\min f(y)=\sum_{i=1}^{p}\left(c_{i}^{T} y+d_{i}\right)\left(e_{i}^{T} y+f_{i}\right) \\
\text { s.t. } \quad y \in Y^{0}=\left\{y \in R^{n} \mid A y \leq b\right\},
\end{array}\right.
$$

(c) The Author(s) 2018. This article is distributed under the terms of the Creative Commons Attribution 4.0 International License (http://creativecommons.org/licenses/by/4.0/), which permits unrestricted use, distribution, and reproduction in any medium, provided you give appropriate credit to the original author(s) and the source, provide a link to the Creative Commons license, and indicate if changes were made. 
where $p \geq 0, c_{i}^{T}=\left(c_{1}, c_{2}, \ldots, c_{n}\right), e_{i}^{T}=\left(e_{1}, e_{2}, \ldots, e_{n}\right) \in R^{n} ; A=\left(a_{j k}\right)_{m \times n}$ is a real $m \times n$ matrix, and $d_{i}, f_{i} \in R, i=1,2, \ldots, n ; b \in R^{m}$. Obviously, the (LMP) can be easily transformed into the (NQP). So, the applications of the (NQP) also include all the applications of the (LMP).

In the last decades, many solution methods have been developed for globally solving the (NQP) and its special forms. For example, branch-and-cut algorithm [8] and branch-and-bound algorithm [9] for box-constrained nonconvex quadratic programs; two efficient algorithms for linear constrained quadratic programming (LCQP) by Cambini et al. [10] and Li et al. [11]; branch-and-reduce approach by Gao et al. [12]; finite branch-and-bound approach by Burer and Vandenbussche [13]; branch-and-bound method based on outer approximation and linear relaxation by Al-Khayyal et al. [14]; two simplicial branch-and-bound algorithms by Linderoth [15] and Raber [16]; branchand-cut algorithm by Sherali et al. [17] and Audet et al. [18], and so on. In addition, a branch-and-bound algorithm for finding a global optimal solution for a nonconvex quadratic program with convex quadratic constraints has been proposed by Cheng Lu et al. [19]; based on the different properties of a quadratic function, five different branch-and-bound approaches for solving the (NQP) have been proposed in Refs. [20-24].

Based on the above-mentioned methods, we will present an accelerating algorithm for effectively solving the (NQP) in this paper. Firstly, we present a new linear relaxation technique, which can be used to construct the linear relaxation programming (LRP) of the (NQP). In addition, we divide the initial feasible region into a number of sub-regions and use deleting techniques to reduce the scope of the investigation. Then, the initial problem (NQP) is converted into a series of linear relaxation programming subproblems. We can prove that the solutions of these subproblems can converge to the global optimal value of the original problem (NQP). Finally, numerical results and comparison with the methods mentioned in recent literature show that our algorithm works as well as or better than those methods.

This paper is organized as follows. In Sect. 2, we describe a new linear relaxation approach for establishing the linear relaxation programming of the (NQP). In Sect. 3, a range deleting technique is given for improving the convergent speed of the proposed algorithm, then a range division and deleting algorithm is proposed and its convergence is proved. Some numerical experiments are reported to show the feasibility and effectiveness of our algorithm in Sect. 4.

\section{New linear relaxation approach}

In this section, we will show how to construct the (LRP) for the (NQP). Let the $i$ th row of the matrix $Q$ be $Q_{i}$, and let $z_{i}=Q_{i} y=\sum_{k=1}^{n} q_{i k} y_{k}, i=1,2, \ldots, n$, we obtain

$$
z=\left(z_{1}, z_{2}, \ldots, z_{n}\right)^{T}=\left(Q_{1} y, Q_{2} y, \ldots, Q_{n} y\right)^{T}=Q y .
$$

By introducing new variables $z_{i}, i=1,2, \ldots, n$, the function $f(y)$ can be expressed as follows:

$$
f(y, z)=c^{T} y+y^{T} z=\sum_{i=1}^{n} c_{i} y_{i}+\sum_{i=1}^{n} y_{i} z_{i}
$$


and the set $D$ is defined as

$$
D=\left\{y \mid A y \leq b, y \in R^{n}\right\} .
$$

We compute the initial variable bounds by solving the following linear programming problems:

$$
\begin{aligned}
& \underline{y}_{i}^{0}=\min _{y \in D} y, \quad \bar{y}_{i}^{0}=\max _{y \in D} y, \quad i=1,2, \ldots, n, \\
& \underline{z}_{i}^{0}=\min _{y \in D} Q_{i} y, \quad \bar{z}_{i}^{0}=\max _{y \in D} Q_{i} y, \quad i=1,2, \ldots, n,
\end{aligned}
$$

and let

$$
\begin{aligned}
& Y^{0}=\left\{y \in R^{n} \mid \underline{y}_{i}^{0} \leq y_{i} \leq \bar{y}_{i}^{0}, i=1,2, \ldots, n\right\}, \\
& Z^{0}=\left\{z \in R^{n} \mid \underline{z}_{i}^{0} \leq z_{i} \leq \bar{z}_{i}^{0}, i=1,2, \ldots, n\right\}, \\
& \Omega^{0}=\left\{(y, z) \in R^{2 n} \mid \underline{y}_{i}^{0} \leq y_{i} \leq \bar{y}_{i}^{0}, \underline{z}_{i}^{0} \leq z_{i} \leq \bar{z}_{i}^{0}, i=1,2, \ldots, n\right\} .
\end{aligned}
$$

Based on the above discussion, we can transform the initial problem into the following equivalent problem (EP):

$$
(\mathrm{EP}):\left\{\begin{aligned}
\min f(y, z)=\sum_{i=1}^{n} c_{i} y_{i}+\sum_{i=1}^{n} y_{i} z_{i} \\
\text { s.t. } \quad A y \leq b, \\
\\
z_{i}=\sum_{k=1}^{n} q_{i k} y_{k}, i=1,2, \ldots, n, \\
(y, z) \in \Omega^{0} .
\end{aligned}\right.
$$

For globally solving the (NQP), the computation of lower bounds for this problem and its subdivided subproblems is the principal operation in the establishment of a branch-andbound procedure. A lower bound on the optimal value of the (NQP) and its subdivided subproblems can be gotten by solving a linear relaxation programming of the (EP), which can be derived by a new linear relaxation approach.

Let

$$
\begin{aligned}
& Y=\left\{y \in R^{n} \mid-\infty \leq \underline{y}_{i} \leq y_{i} \leq \bar{y}_{i} \leq+\infty, i=1,2, \ldots, n\right\} \subseteq Y^{0}, \\
& Z=\left\{z \in R^{n} \mid-\infty \leq \underline{z}_{i} \leq z_{i} \leq \bar{z}_{i} \leq+\infty, i=1,2, \ldots, n\right\} \subseteq Z^{0}
\end{aligned}
$$

for any $y \in Y, z \in Z, i \in\{1,2, \ldots, n\}$, define the functions

$$
\begin{aligned}
& \varphi\left(y_{i}\right)=y_{i}^{2}, \quad \varphi^{l}\left(y_{i}\right)=\left(\underline{y}_{i}+\bar{y}_{i}\right) y_{i}-\frac{\left(\underline{y}_{i}+\bar{y}_{i}\right)^{2}}{4}, \\
& \varphi^{u}\left(y_{i}\right)=\left(\underline{y}_{i}+\bar{y}_{i}\right) y_{i}-\underline{y}_{i} \bar{y}_{i}, \\
& \varphi\left(z_{i}\right)=z_{i}^{2}, \quad \varphi^{l}\left(z_{i}\right)=\left(\underline{z}_{i}+\bar{z}_{i}\right) z_{i}-\frac{\left(\underline{z}_{i}+\bar{z}_{i}\right)^{2}}{4}, \\
& \varphi^{u}\left(z_{i}\right)=\left(\underline{z}_{i}+\bar{z}_{i}\right) z_{i}-\underline{z}_{i} \bar{z}_{i}, \quad \varphi\left(y_{i}, z_{i}\right)=y_{i} z_{i},
\end{aligned}
$$




$$
\begin{aligned}
\varphi^{l}\left(y_{i}, z_{i}\right) & =\frac{1}{2}\left[\left(\underline{y}_{i}+\bar{y}_{i}\right) z_{i}+\left(\underline{z}_{i}+\bar{z}_{i}\right) y_{i}-\frac{\left(\underline{y}_{i}+\bar{y}_{i}+\underline{z}_{i}+\bar{z}_{i}\right)^{2}}{4}+\underline{y}_{i} \bar{y}_{i}+\underline{z}_{i} \bar{z}_{i}\right] \\
\varphi^{u}\left(y_{i}, z_{i}\right) & =\frac{1}{2}\left[\left(\underline{y}_{i}+\bar{y}_{i}\right) z_{i}+\left(\underline{z}_{i}+\bar{z}_{i}\right) y_{i}-\left(\underline{y}_{i}+\underline{z}_{i}\right)\left(\bar{y}_{i}+\bar{z}_{i}\right)+\frac{\left(\underline{y}_{i}+\bar{y}_{i}\right)^{2}}{4}+\frac{\left(\underline{z}_{i}+\bar{z}_{i}\right)^{2}}{4}\right] .
\end{aligned}
$$

Since $\varphi\left(y_{i}\right)=y_{i}^{2}$ is a convex function of $y_{i}$ over $\left[y_{i}, \overline{y_{i}}\right]$, its affine concave envelope is $\varphi^{u}\left(y_{i}\right)=\left(y_{i}+\overline{y_{i}}\right) y_{i}-y_{i} \overline{y_{i}}$. Moreover, the tangential supporting function for $\varphi\left(y_{i}\right)$ is parallel with the $\varphi^{u}\left(y_{i}\right)$, thus the point of tangential support will occur at $\frac{y_{i}+\overline{y_{i}}}{2}$, and the corresponding tangential supporting function is $\varphi^{l}\left(y_{i}\right)=\left(\underline{y_{i}}+\overline{y_{i}}\right) y_{i}-\frac{\left(y_{i}+\bar{y}_{i}\right)^{2}}{4}$. Hence, by the geometric property of the function $\varphi\left(y_{i}\right)$, we have

$$
\left.\varphi^{l}\left(y_{i}\right)=\left(\underline{y_{i}}+\overline{y_{i}}\right) y_{i}-\frac{\left(y_{i}+\overline{y_{i}}\right)^{2}}{4} \leq y_{i}^{2} \leq \underline{\left(y_{i}\right.}+\overline{y_{i}}\right) y_{i}-\underline{y_{i}} \overline{y_{i}}=\varphi^{u}\left(y_{i}\right) .
$$

Similarly, it follows that

$$
\varphi^{l}\left(z_{i}\right) \leq \varphi\left(z_{i}\right) \leq \varphi^{u}\left(z_{i}\right)
$$

Furthermore, assume that $\left(y_{i}+z_{i}\right)$ is a single variable, then $\left(y_{i}+z_{i}\right)^{2}$ is a convex function about the univariate variable $\left(y_{i}+z_{i}\right)$ over the interval $\left[y_{i}+\underline{z_{i}}, \overline{y_{i}}+\overline{z_{i}}\right]$. By the conclusion above, we have

$$
\left(\underline{y_{i}}+\underline{z_{i}}+\overline{y_{i}}+\overline{z_{i}}\right)\left(y_{i}+z_{i}\right)-\frac{\left(y_{i}+\underline{z_{i}}+\overline{y_{i}}+\overline{z_{i}}\right)^{2}}{4} \leq\left(y_{i}+z_{i}\right)^{2}
$$

and

$$
\left(y_{i}+z_{i}\right)^{2} \leq\left(\underline{y_{i}}+\underline{z_{i}}+\overline{y_{i}}+\overline{z_{i}}\right)\left(y_{i}+z_{i}\right)-\left(\underline{y_{i}}+\underline{z_{i}}\right)\left(\overline{y_{i}}+\overline{z_{i}}\right) .
$$

By (1)-(3), we can obtain

$$
\begin{aligned}
\varphi\left(y_{i}, z_{i}\right)= & \frac{1}{2}\left[\left(y_{i}+z_{i}\right)^{2}-y_{i}^{2}-z_{i}^{2}\right] \\
\geq & \frac{1}{2}\left[\left(\underline{y}_{i}+\underline{z}_{i}+\bar{y}_{i}+\bar{z}_{i}\right)\left(y_{i}+z_{i}\right)-\frac{\left(\underline{y}_{i}+\underline{z}_{i}+\bar{y}_{i}+\bar{z}_{i}\right)^{2}}{4}\right] \\
& -\frac{1}{2}\left[\left(\underline{y}_{i}+\bar{y}_{i}\right) y_{i}-\underline{y}_{i} \bar{y}_{i}+\left(\underline{z}_{i}+\bar{z}_{i}\right) z_{i}-\underline{z}_{i} \bar{z}_{i}\right] \\
= & \frac{1}{2}\left[\left(\underline{y}_{i}+\bar{y}_{i}\right) z_{i}+\left(\underline{z}_{i}+\bar{z}_{i}\right) y_{i}-\frac{\left(\underline{y}_{i}+\underline{z}_{i}+\bar{y}_{i}+\bar{z}_{i}\right)^{2}}{4}+\underline{y}_{i} \bar{y}_{i}+\underline{z}_{i} \bar{z}_{i}\right] \\
= & \varphi^{l}\left(y_{i}, z_{i}\right),
\end{aligned}
$$

and

$$
\begin{aligned}
\varphi\left(y_{i}, z_{i}\right) & =\frac{1}{2}\left[\left(y_{i}+z_{i}\right)^{2}-y_{i}^{2}-y_{k}^{2}\right] \\
& \leq \frac{1}{2}\left[\left(\underline{y}_{i}+\underline{z}_{i}+\bar{y}_{i}+\bar{z}_{i}\right)\left(y_{i}+z_{i}\right)-\left(\underline{y}_{i}+\underline{z}_{i}\right)\left(\bar{y}_{i}+\bar{z}_{i}\right)\right]
\end{aligned}
$$




$$
\begin{aligned}
& -\frac{1}{2}\left[\left(\underline{y}_{i}+\bar{y}_{i}\right) y_{i}-\frac{\left(\underline{y}_{i}+\bar{y}_{i}\right)^{2}}{4}+\left(\underline{z}_{i}+\bar{z}_{i}\right) z_{i}-\frac{\left(\underline{z}_{i}+\bar{z}_{i}\right)^{2}}{4}\right] \\
= & \frac{1}{2}\left[\left(\underline{y}_{i}+\bar{y}_{i}\right) z_{i}+\left(\underline{z}_{i}+\bar{z}_{i}\right) y_{i}-\left(\underline{y}_{i}+\underline{z}_{i}\right)\left(\bar{y}_{i}+\bar{z}_{i}\right)+\frac{\left(\underline{y}_{i}+\bar{y}_{i}\right)^{2}}{4}+\frac{\left(\underline{z}_{i}+\bar{z}_{i}\right)^{2}}{4}\right] \\
= & \varphi^{u}\left(y_{i}, z_{i}\right) .
\end{aligned}
$$

Hence, we have

$$
\varphi^{l}\left(y_{i}, z_{i}\right) \leq \varphi\left(y_{i}, z_{i}\right) \leq \varphi^{u}\left(y_{i}, z_{i}\right)
$$

Furthermore, we define the difference functions as follows:

$$
\begin{aligned}
& \triangle\left(y_{i}\right)=\varphi\left(y_{i}\right)-\varphi^{l}\left(y_{i}\right), \quad \nabla\left(y_{i}\right)=\varphi^{u}\left(y_{i}\right)-\varphi\left(y_{i}\right), \\
& \triangle\left(z_{i}\right)=\varphi\left(z_{i}\right)-\varphi^{l}\left(z_{i}\right), \quad \nabla\left(z_{i}\right)=\varphi^{u}\left(z_{i}\right)-\varphi\left(z_{i}\right), \\
& \triangle\left(y_{i}, z_{i}\right)=\varphi\left(y_{i}, z_{i}\right)-\varphi^{l}\left(y_{i}, z_{i}\right), \quad \nabla\left(y_{i}, z_{i}\right)=\varphi^{u}\left(y_{i}, z_{i}\right)-\varphi\left(y_{i}, z_{i}\right) .
\end{aligned}
$$

Since $\Delta\left(y_{i}\right)$ is convex about $y_{i}$, for any $y_{i} \in\left[\underline{y_{i}}, \overline{y_{i}}\right]$, it follows that $\triangle\left(y_{i}\right)$ can attain the maximum at the point $\underline{y_{i}}$ or $\overline{y_{i}}$. Then

$$
\max _{y_{i} \in\left[\underline{\left.y_{i}, \overline{y_{i}}\right]}\right.} \Delta\left(y_{i}\right)=\varphi\left(\overline{y_{i}}\right)-\varphi^{l}\left(\overline{y_{i}}\right)=\varphi\left(\underline{y_{i}}\right)-\varphi^{l}\left(\underline{y_{i}}\right)=\frac{\left(\overline{y_{i}}-\underline{y_{i}}\right)^{2}}{4} .
$$

On the other hand, since $\nabla\left(y_{i}\right)$ is concave about $y_{i}$, for any $y_{i} \in\left[y_{i}, \overline{y_{i}}\right], \nabla\left(y_{i}\right)$ can attain the maximum at the point $\frac{y_{i}+\overline{y_{i}}}{2}$, i.e.,

$$
\max _{y_{i} \in\left[\underline{\left.y_{i}, \overline{y_{i}}\right]}\right.} \nabla\left(y_{i}\right)=\varphi^{u}\left(\frac{\underline{y_{i}}+\overline{y_{i}}}{2}\right)-\varphi\left(\frac{\underline{y_{i}}+\overline{y_{i}}}{2}\right)=\frac{\left(\overline{y_{i}}-\underline{y_{i}}\right)^{2}}{4} .
$$

So, we have

$$
\max _{y_{i} \in\left[\underline{y}_{i} \bar{y}_{i}\right]} \Delta\left(y_{i}\right)=\max _{y_{i} \in\left[\underline{y}_{i} \bar{y}_{i}\right]} \nabla\left(y_{i}\right) \rightarrow 0, \quad \text { as }\left|\bar{y}_{i}-\underline{y}_{i}\right| \rightarrow 0
$$

that is, $\triangle\left(y_{i}\right), \nabla\left(y_{i}\right) \rightarrow 0$, as $\|\bar{y}-\underline{y}\| \rightarrow 0$.

Similarly, we can prove that

$$
\max _{z_{i} \in\left[\underline{z}_{i}, \bar{z}_{i}\right]} \Delta\left(z_{i}\right)=\max _{z_{i} \in\left[\underline{z}_{i}, \bar{z}_{i}\right]} \nabla\left(z_{i}\right) \rightarrow 0, \quad \text { as }\left|\bar{z}_{i}-\underline{z}_{i}\right| \rightarrow 0,
$$

and $\triangle\left(z_{i}\right), \nabla\left(z_{i}\right) \rightarrow 0$, as $\|\bar{z}-\underline{z}\| \rightarrow 0$.

Define

$$
\begin{aligned}
& \Delta\left(y_{i}+z_{i}\right)=\left(y_{i}+z_{i}\right)^{2}-\left[\left(\underline{y}_{i}+\bar{y}_{i}+\underline{z}_{i}+\bar{z}_{i}\right)\left(y_{i}+z_{i}\right)-\frac{\left(\underline{y}_{i}+\bar{y}_{i}+\underline{z}_{i}+\bar{z}_{i}\right)^{2}}{4}\right], \\
& \nabla\left(y_{i}+z_{i}\right)=\left(\underline{y}_{i}+\bar{y}_{i}+\underline{z}_{i}+\bar{z}_{i}\right)\left(y_{i}+z_{i}\right)-\left(\underline{y}_{i}+\underline{z}_{i}\right)\left(\bar{y}_{i}+\bar{z}_{i}\right)-\left(y_{i}+z_{i}\right)^{2} .
\end{aligned}
$$


Using the similar method, we can get the following conclusions:

$$
\begin{aligned}
& \max _{\left(y_{i}+z_{i}\right) \in\left[\left(\underline{y}_{i}+\underline{z}_{i}\right),\left(\bar{y}_{i}+\bar{z}_{i}\right)\right]} \Delta\left(y_{i}+z_{i}\right) \\
& =\max _{\left(y_{i}+z_{i}\right) \in\left[\left(\underline{y}_{i}+\underline{z}_{i}\right),\left(\bar{y}_{i}+\bar{z}_{i}\right)\right]} \nabla\left(y_{i}+z_{i}\right) \rightarrow 0, \quad \text { as }\|\bar{y}-\underline{y}\| \rightarrow 0,\|\bar{z}-\underline{z}\| \rightarrow 0 .
\end{aligned}
$$

Since

$$
\begin{aligned}
\triangle\left(y_{i}, z_{i}\right)= & \varphi\left(y_{i}, z_{i}\right)-\varphi^{l}\left(y_{i}, z_{i}\right) \\
= & y_{i} z_{i}-\frac{1}{2}\left[\left(\underline{y}_{i}+\bar{y}_{i}\right) z_{i}+\left(\underline{z}_{i}+\bar{z}_{i}\right) y_{i}-\frac{\left(y_{i}+\bar{y}_{i}+\underline{z}_{i}+\bar{z}_{i}\right)^{2}}{4}+\underline{y}_{i} \bar{y}_{i}+\underline{z}_{i} \bar{z}_{i}\right] \\
= & \frac{1}{2}\left[\left(y_{i}+z_{i}\right)^{2}-y_{i}^{2}-y_{k}^{2}\right] \\
& -\frac{1}{2}\left[\left(\underline{y}_{i}+\bar{y}_{i}\right) z_{i}+\left(\underline{z}_{i}+\bar{z}_{i}\right) y_{i}-\frac{\left(\underline{y}_{i}+\bar{y}_{i}+\underline{z}_{i}+\bar{z}_{i}\right)^{2}}{4}+\underline{y}_{i} \bar{y}_{i}+\underline{z}_{i} \bar{z}_{i}\right] \\
= & \frac{1}{2}\left[\left(y_{i}+z_{i}\right)^{2}-\left(\left(\underline{y}_{i}+\bar{y}_{i}+\underline{z}_{i}+\bar{z}_{i}\right)\left(y_{i}+z_{i}\right)-\frac{\left(\underline{y}_{i}+\bar{y}_{i}+\underline{z}_{i}+\bar{z}_{i}\right)^{2}}{4}\right)\right] \\
& +\frac{1}{2}\left[\left(\left(\underline{y}_{i}+\bar{y}_{i}\right) y_{i}-\underline{y}_{i} \bar{y}_{i}-y_{i}^{2}\right)+\left(\left(\underline{z}_{i}+\bar{z}_{i}\right) z_{i}-\underline{z}_{i} \bar{z}_{i}-z_{i}^{2}\right)\right] \\
= & \frac{1}{2} \triangle\left(y_{i}+z_{i}\right)+\frac{1}{2} \nabla\left(y_{i}\right)+\frac{1}{2} \nabla\left(z_{i}\right) \\
\leq & \frac{1}{2} \max _{\left.\left(y_{i}+z_{i}\right) \in\left[\underline{y}_{i}+\underline{z}_{i}\right),\left(\bar{y}_{i}+\bar{z}_{i}\right)\right]} \triangle\left(y_{i}+z_{i}\right)+\frac{1}{2} \max _{y_{i} \in\left[\underline{y}_{i} \bar{y}_{i}\right]} \nabla\left(y_{i}\right)+\frac{1}{2} \max _{z_{i} \in\left[\underline{z}_{i} \bar{z}_{i}\right]} \nabla\left(z_{i}\right),
\end{aligned}
$$

by (5)-(7), we can get that

$$
\triangle\left(y_{i}, z_{i}\right) \rightarrow 0, \quad \text { as }\|\bar{y}-\underline{y}\| \rightarrow 0,\|\bar{z}-\underline{z}\| \rightarrow 0 .
$$

Similarly, we can prove that $\nabla\left(y_{i}, z_{i}\right) \rightarrow 0$, as $\|\bar{y}-\underline{y}\| \rightarrow 0,\|\bar{z}-\underline{z}\| \rightarrow 0$.

For convenience, without loss of generality, for any $y \in Y \subseteq Y^{0}, z \in Z \subseteq Z^{0}$, define

$$
f^{L}(y, z)=\sum_{i=1}^{n} c_{i} y_{i}+\sum_{i=1}^{n} \varphi^{l}\left(y_{i}, z_{i}\right) .
$$

By utilizing the convexity of a univariate quadratic function, we establish an effective method for generating the linear underestimation and linear overestimation of the functions $y_{i}^{2}, z_{i}^{2}$, and $y_{i} z_{i}$, respectively. By the conclusions above, the linear relaxation underestimation function $f^{L}(y, z)$ of the function $f(y, z)$ for the (EP) can be established. Thus, by the former discussions, we can construct the corresponding approximation linear relaxation programming (LRP) of the (EP) as follows:

$(\mathrm{LRP}): \begin{cases}\min f^{L}(y, z)=\sum_{i=1}^{n} c_{i} y_{i}+\sum_{i=1}^{n} \varphi^{l}\left(y_{i}, z_{i}\right) \\ \text { s.t. } \quad A y \leq b, \\ \\ z_{i}=\sum_{k=1}^{n} q_{i k} y_{k}, i=1,2, \ldots, n, \\ (y, z) \in \Omega^{0} .\end{cases}$ 
By (4), it is obvious that

$$
y_{i} z_{i}-\varphi^{l}\left(y_{i}, z_{i}\right) \geq 0
$$

then

$$
\begin{aligned}
& f(y, z)-f^{L}(y, z) \\
& \quad=\sum_{k=1}^{n} c_{i} y_{i}+\sum_{k=1}^{n} y_{i} z_{i}-\left[\sum_{k=1}^{n} c_{i} y_{i}+\sum_{k=1}^{n} \varphi^{l}\left(y_{i}, z_{i}\right)\right] \\
& \quad=\sum_{k=1}^{n}\left[y_{i} z_{i}-\varphi^{l}\left(y_{i}, z_{i}\right)\right] \\
& \quad \geq 0 .
\end{aligned}
$$

Thus, we have $f(y, z)-f^{L}(y, z) \geq 0$, i.e., $f(y, z) \geq f^{L}(y, z)$.

Furthermore, we have

$$
\begin{aligned}
f(y, z)-f^{L}(y, z) & =\sum_{k=1}^{n}\left[y_{i} z_{i}-\varphi^{l}\left(y_{i}, z_{i}\right)\right] \\
& =\sum_{k=1}^{n} \triangle\left(y_{i}, z_{i}\right) .
\end{aligned}
$$

By (8), we have that $\triangle\left(y_{i}, z_{i}\right) \rightarrow 0$, as $\|\bar{y}-y\| \rightarrow 0,\|\bar{z}-\underline{z}\| \rightarrow 0$.

Therefore, we have that

$$
f(y, z)-f^{L}(y, z) \rightarrow 0 \quad \text { as }\|\bar{y}-\underline{y}\| \rightarrow 0,\|\bar{z}-\underline{z}\| \rightarrow 0
$$

Remark 1 From above, we only need to solve the (LRP) instead of solving the (EP) to obtain the lower and upper bounds of the optimal value in problem (NQP).

Remark 2 Based on the construction of the (LRP), each feasible point of the (NQP) in the sub-range $\Omega$ is also feasible to LRP, and the global minimum value of LRP is not more than that of the (NQP) in the sub-range $\Omega$. Thus, the (LRP) can provide a valid lower bound for the global optimum value of problem NQP in the sub-range $\Omega$.

Remark 3 The conclusions above ensure that the linear relaxation programming LRP can infinitely approximate the (NQP), as $\|Y\| \rightarrow 0$ (obviously, $\|Z\| \rightarrow 0$ ), this will guarantee the global convergence of the proposed algorithm.

\section{Accelerating branch-and-bound algorithm and its convergence}

Now we establish an accelerating branch-and-bound algorithm based upon the former linear relaxation approach for globally solving the (NQP). We will introduce the algorithm process at first and then give the convergence analysis of the algorithm. 


\subsection{Branching process}

The critical operation of the branching process is iteratively subdividing the initial range $\Omega^{0}$ into some sub-ranges, each sub-range denoted by $\Omega$ is concerned with a node of the branch and bound, such that any infinite iterative sequence of partition sets shrinks to a singleton. Here, we will adopt a standard range bisection approach, which is adequate to ensuring global convergence of the proposed algorithm. Detailed process is described as follows.

For any selected sub-range $\Omega^{\prime} \in \Omega^{0}, Y^{\prime}=\left[y^{\prime}, \bar{y}^{\prime}\right] \subseteq Y^{0}$, let $q \in \arg \max \left\{\bar{y}_{i}^{\prime}-\underline{y}_{i}^{\prime}: i=\right.$ $1,2, \ldots, n\}$, and $y_{m}=\left(\underline{y}_{q}^{\prime}+\bar{y}_{q}^{\prime}\right) / 2$, then divide $\Omega^{\prime}$ into two sub-ranges $\Omega_{1}^{\prime}$ and $\Omega_{2}^{\prime}$ by subdividing the interval $\left[y_{q}^{\prime}, \bar{y}_{q}^{\prime}\right]$ into two subintervals $\left[\underline{y}_{q}^{\prime}, y_{m}\right]$ and $\left[y_{m}, \bar{y}_{q}^{\prime}\right]$.

From the above branching operation, we can notice that the intervals $\left[\underline{z}^{\prime}, \bar{z}^{\prime}\right]$ of $z$ will never be partitioned by the branching processes. Hence, these branching operations only occur in a space of dimension $n$, i.e., the proposed algorithm economizes the required computations.

\subsection{Range deleting technique}

At the $k$ th iteration of the algorithm, for any rectangle $\Omega^{k} \subseteq \Omega^{0}$, we want to check whether $\Omega^{k}$ contains a global optimal solution of the $(\mathrm{EP})\left(\Omega^{0}\right)$, where

$$
\Omega^{k}=\Omega_{1}^{k} \times \Omega_{2}^{k} \times \cdots \times \Omega_{q-1}^{k} \times \Omega_{q}^{k} \times \Omega_{q+1}^{k} \times \cdots \times \Omega_{n}^{k},
$$

with

$$
\Omega_{q}^{k}=\left\{\left(y_{q}, z_{q}\right) \in R^{2} \mid \underline{y}_{q}^{k} \leq y_{q}^{k} \leq \bar{y}_{q}^{k}, z_{q}^{0} \leq z_{q}^{0} \leq \bar{z}_{q}^{0}\right\}
$$

Theorem 3.1 Assume that $\bar{f}$ is a known upper bound of the optimal value $v$ of the (EP), for any sub-range $\Omega^{k} \subseteq \Omega^{0}$, the following conclusions hold: (i) If $E L B^{k}>\bar{f}$, then there is no global optimal solution for the (EP) over $\Omega^{k}$; (ii) If $E L B^{k} \leq \bar{f}$, then we have: for any $\tau \in\{1,2, \ldots, n\}$, if $c_{\tau}>0$, then the sub-range $\bar{\Omega}^{k}$ does not contain any global optimal solution of the (EP); else if $c_{\tau}<0$, then the sub-range $\overline{\bar{\Omega}}^{k}$ does not contain any global optimal solution of the $(E P)$, where

$$
\begin{aligned}
& E L B^{k}=\sum_{i=1}^{n} \min \left\{c_{i} \underline{y}_{i}^{k}, c_{i} \bar{y}_{i}^{k}\right\}+\sum_{i=1}^{n} \min \left\{\underline{y}_{i}^{k} \underline{z}_{i}^{0}, \underline{y}_{i}^{k} \bar{z}_{i}^{0}, \bar{y}_{i}^{k} \underline{z}_{i}^{0}, \bar{y}_{i}^{k} \bar{z}_{i}^{0}\right\}, \\
& \rho_{\tau}^{k}=\frac{\bar{f}-R L B^{k}+\min \left\{c_{\tau} \underline{y}_{-\tau}^{k}, c_{\tau} \bar{y}_{\tau}^{k}\right\}}{c_{\tau}}, \quad \tau=1, \ldots, n, \\
& \bar{\Omega}^{k}=\Omega_{1}^{k} \times \Omega_{2}^{k} \times \cdots \times \Omega_{\tau-1}^{k} \times \bar{\Omega}_{\tau}^{k} \times \Omega_{\tau+1}^{k} \times \cdots \times \Omega_{n}^{k}, \\
& \overline{\bar{\Omega}}^{k}=\Omega_{1}^{k} \times \Omega_{2}^{k} \times \cdots \times \Omega_{\tau-1}^{k} \times \overline{\bar{\Omega}}_{\tau}^{k} \times \Omega_{\tau+1}^{k} \times \cdots \times \Omega_{n}^{k},
\end{aligned}
$$

with

$$
\begin{aligned}
& \bar{\Omega}_{\tau}^{k}=\left\{\left(y_{\tau}, z_{\tau}\right) \in R^{2} \mid \rho_{\tau}^{k} \leq y_{\tau}^{k} \leq \bar{y}_{\tau}^{k}, z_{\tau}^{0} \leq z_{\tau}^{0} \leq \bar{z}_{\tau}^{0}\right\} \cap \Omega_{\tau}^{k}, \\
& \bar{\Omega}_{\tau}^{k}=\left\{\left(y_{\tau}, z_{\tau}\right) \in R^{2} \mid y_{\tau}^{k} \leq y_{\tau}^{k} \leq \rho_{\tau}^{k}, \underline{z}_{\tau}^{0} \leq z_{\tau}^{0} \leq \bar{z}_{\tau}^{0}\right\} \cap \Omega_{\tau}^{k} .
\end{aligned}
$$


Proof (i) If $E L B^{k}>\bar{f}$, then for all $(y, z) \in \Omega^{k}$,

$$
\begin{aligned}
f(y, z) & =\sum_{i=1}^{n} c_{i} y_{i}+\sum_{i=1}^{n} y_{i} z_{i} \\
& \geq \sum_{i=1}^{n} \min \left\{c_{i} \underline{y}_{i}^{k}, c_{i} \bar{y}_{i}^{k}\right\}+\sum_{i=1}^{n} \min \left\{\underline{y}_{i}^{k} \underline{z}_{i}^{0}, \underline{y}_{i}^{k} \bar{z}_{i}^{0}, \bar{y}_{i}^{k} \underline{z}_{i}^{0}, \bar{y}_{i}^{k} \bar{z}_{i}^{0}\right\} \\
& =E L B^{k}>\bar{f} .
\end{aligned}
$$

Therefore, there is no global optimal solution for the (EP) over $\Omega^{k}$.

(ii) If $E L B^{k} \leq \bar{f}$, for any $\tau \in\{1,2, \ldots, n\}$, then consider the following two cases.

Case 1: If $c_{\tau}>0$, for all $(y, z) \in \bar{\Omega}^{k}$, we have $y_{\tau}>\rho_{\tau}$, i.e., $c_{\tau} y_{\tau}>\bar{f}-E L B^{k}+\min \left\{c_{\tau} \underline{y}_{\tau}^{k}, c_{\tau} \bar{y}_{\tau}^{k}\right\}$.

Furthermore, we can get that

$$
\begin{aligned}
f(y, z)= & \sum_{i=1, i \neq \tau}^{n} c_{i} y_{i}+c_{\tau} y_{\tau}+\sum_{i=1}^{n} y_{i} z_{i} \\
\geq & \sum_{i=1, i \neq \tau}^{n} \min \left\{c_{i} y_{i}^{k}, c_{i} \bar{y}_{i}^{k}\right\}+c_{\tau} y_{\tau}+\sum_{i=1}^{n} \min \left\{\underline{y}_{i}^{k} \underline{z}_{i}^{0}, \underline{y}_{i}^{k} \bar{z}_{i}^{0}, \bar{y}_{i}^{k} \underline{z}_{i}^{0}, \bar{y}_{i}^{k} \bar{z}_{i}^{0}\right\} \\
> & \sum_{i=1, i \neq \tau}^{n} \min \left\{c_{i} \underline{y}_{i}^{k}, c_{i} \bar{y}_{i}^{k}\right\}+\sum_{i=1}^{n} \min \left\{\underline{y}_{i}^{k} \underline{z}_{i}^{0}, \underline{y}_{i}^{k} \bar{z}_{i}^{0}, \bar{y}_{i}^{k} \underline{z}_{i}^{0}, \bar{y}_{i}^{k} \bar{z}_{i}^{0}\right\}+\bar{f} \\
& -E L B^{k}+\min \left\{c_{\tau} \underline{y}_{-}^{k}, c_{\tau} \bar{y}_{\tau}^{k}\right\} \\
= & E L B^{k}+\bar{f}-E L B^{k} \\
= & \bar{f} .
\end{aligned}
$$

Thus, we have

$$
f(y, z)>\bar{f}
$$

Therefore, the range $\bar{\Omega}^{k}$ does not contain any global optimal solution of the (EP).

Case 2: If $c_{\tau}<0$, then for any $(y, z) \in \overline{\bar{\Omega}}^{k}$, we have $y_{\tau}<\rho_{\tau}$, i.e., $c_{\tau} y_{\tau}>\bar{f}-E L B^{k}+$ $\min \left\{c_{\tau} \underline{y}_{\tau}^{k}, c_{\tau} \bar{y}_{\tau}^{k}\right\}$.

Similar to the proof of Case 1, we can get that

$$
f(y, z)>\bar{f}
$$

thus the range $\overline{\bar{\Omega}}^{k}$ does not contain any global optimal solution of the (EP). Thus, the proof is completed.

According to Theorem 3.1, we can construct the following range reduction technique to reject the whole investigated sub-range $\Omega^{k}$ or delete a part of the investigated sub-range $\Omega^{k}$ which does not contain any global optimal solution of the (EP).

\section{Range deleting algorithm}

Calculate $E L B^{k}$, if $E L B^{k}>\bar{f}$, then let $\Omega^{k}=\emptyset$. Otherwise, for each $\tau \in\{1, \ldots, n\}$, if $c_{\tau}>0$ and $\rho_{\tau}^{k}<\bar{y}_{\tau}^{k}$, then let $\bar{y}_{\tau}^{k}=\rho_{\tau}^{k}$ and $\bar{\Omega}^{k}$ with $\bar{\Omega}_{\tau}^{k}=\left\{\left(y_{\tau}, z_{\tau}\right) \in R^{2} \mid \underline{y}_{\tau}^{k} \leq y_{\tau}^{k} \leq \bar{y}_{\tau}^{k}, \underline{z}_{\tau}^{0} \leq z_{\tau}^{0} \leq \bar{z}_{\tau}^{0}\right\}$; 
else if $c_{\tau}<0$ and $\rho_{\tau}^{k}>y_{\tau}^{k}$, then let $\underline{y}_{\tau}^{k}=\rho_{\tau}^{k}$ and $\bar{\Omega}^{k}$ with $\bar{\Omega}_{\tau}^{k}=\left\{\left(y_{\tau}, z_{\tau}\right) \in R^{2} \mid y_{\tau}^{k} \leq y_{\tau}^{k} \leq \bar{y}_{\tau}^{k}, \underline{z}_{\tau}^{0} \leq\right.$ $\left.z_{\tau}^{0} \leq \bar{z}_{\tau}^{0}\right\}$

\subsection{Branch-and-bound algorithm}

Assume that we have gotten the set of active nodes $\Lambda_{k}$ at the $k$ th iteration of the algorithm, and each node is associated with a sub-range $\Omega \subseteq \Omega^{0}$ for all $\Omega \in \Lambda_{k}$. For each sub-range $\Omega$, use the proposed range deleting technique to compress the sub-range $\Omega$, still denote the remaining sub-range by $\Omega$, and obtain a lower bound $L B(\Omega)$ of the optimum value of the (NQP) by solving the (LRP) in $\Omega$.

Let $f_{r}(\Omega)$ and $\left(y_{r}(\Omega), z_{r}(\Omega)\right)$ be the optimal value and the optimal solution of the (LRP) over the sub-range $\Omega$, respectively. Combining the former linear relaxation method, branching process, and range deleting technique, the basic steps of the proposed accelerating algorithm for globally solving the (NQP) may be stated as follows.

\section{Algorithm statement}

Initialization step. Let the initial iteration counter $k:=0$, the initial set of active nodes $\Lambda_{0}=\left\{\Omega^{0}\right\}$, the initial upper bound $\bar{f}=+\infty$, the convergent error $\varepsilon>0$, and the initial collection of feasible points $F:=\emptyset$.

Solve the (LRP) over $\Omega^{0}$, obtain its optimal solution $y^{0}:=y_{r}\left(\Omega^{0}\right), z^{0}:=z_{r}\left(\Omega^{0}\right)$ and optimal objective function value $L B_{0}:=f_{r}\left(\Omega^{0}\right)$. If $\left(y^{0}, z^{0}\right)$ is feasible to the (NQP), then let $\bar{f}=f\left(y^{0}, z^{0}\right)$ and $F=F \cup\left\{\left(y^{0}, z^{0}\right)\right\}$. If $\bar{f}-L B_{0} \leq \varepsilon$, then algorithm stops with $\left(y^{0}, z^{0}\right)$ as an $\varepsilon$-global optimum solution for the (NQP). Otherwise, proceed with the following Range deleting step.

Range deleting step. For each sub-range $\Omega$, use the proposed range deleting technique in Sect. 3.2 to contract each sub-range $\Omega$ and still denote the remaining sub-range by $\Omega$.

Range division step. Apply the presented range division approach to subdivide $\Omega^{k}$ into two new sub-ranges, and denote the collection of new subdivided sub-ranges by $\widehat{\Omega}^{k}$.

Renewing the lower and upper bound step. If $\widehat{\Omega}^{k} \neq \emptyset$ for each $\Omega \in \widehat{\Omega}^{k}$, then solve the $\operatorname{LRP}(\Omega)$ to get $f_{r}(\Omega)$ and $\left(y_{r}(\Omega), z_{r}(\Omega)\right)$. Let $L B(\Omega):=f_{r}(\Omega)$, if $L B(\Omega)>\bar{f}$, set $\widehat{\Omega}^{k}:=\widehat{\Omega}^{k} \backslash \Omega$. The residual subdivided set is now $\Lambda_{k}:=\left(\Lambda_{k} \backslash \Omega^{k}\right) \cup \widehat{\Omega}^{k}$, and renew the lower bound $L B_{k}:=\inf _{\Omega \in \Lambda_{k}} L B(\Omega)$.

If the midpoint $y_{\text {mid }}$ of $Y$ is feasible to problem (NQP), set $z_{\text {mid }}=Q y_{\text {mid }}$, then let $F:=$ $F \cup\left\{\left(y_{\text {mid }}, z_{\text {mid }}\right)\right\}$. Furthermore, if $y_{r}(\Omega)$ is feasible to problem (NQP), then let $F:=F \cup$ $\left\{\left(y_{r}(\Omega)\right), z_{r}(\Omega)\right\}$.

If $F \neq \emptyset$, renew the upper bound $\bar{f}:=\min _{(y, z) \in F} f(y, z)$, and denote the known best feasible solution by $\left(y_{\text {best }}, z_{\text {best }}\right):=\operatorname{argmin}_{(y, z) \in F} f(y, z)$.

Termination checking step. If $\bar{f}-L B_{k} \leq \varepsilon$, the algorithm ends with $\bar{f}$ and $y_{\text {best }}$ as the $\varepsilon$-global optimum value and a global optimal solution of problem (NQP). Otherwise, set $k:=k+1$ and pick out an active node $\Omega^{k+1}$ satisfying $\Omega^{k+1}=\operatorname{argmin}_{\Omega \in \Lambda_{k}} L B(\Omega)$, and return to Range deleting step.

\subsection{Convergence analysis}

In this subsection, the convergence of this algorithm is discussed as follows.

Theorem 3.2 Suppose that the feasible region $D$ of the $(N Q P)$ is nonempty, then the above algorithm either terminates finitely with a global optimum solution of the (NQP), or generates an infinite sequence $\left\{y^{k}\right\}$ of which any accumulation point will be a global optimal solution of the (NQP). 
Proof If the algorithm terminates finitely, it terminates at the $k$ th iteration, where $k \geq 0$. Upon termination, by steps in the algorithm, we get that $\bar{f}-L B_{k} \leq \varepsilon$. By the first five steps in the algorithm, there exists a feasible solution $y^{*}$ for the (NQP), which satisfies that $\bar{f}=f\left(y^{*}\right)$, and $f\left(y^{*}\right)-L B_{k} \leq \varepsilon$. According to the algorithm, we have that $L B_{k} \leq v$. Since $y^{*}$ is a feasible solution of the (NQP), we have $f\left(y^{*}\right) \geq v$. Combining the above inequalities, we get that $v \leq f\left(y^{*}\right) \leq L B_{k}+\varepsilon \leq v+\varepsilon$, i.e., $v \leq f\left(y^{*}\right) \leq v+\varepsilon$. Therefore, $y^{*}$ is a global $\varepsilon$-optimum solution for the (NQP).

If the algorithm is infinite, according to the algorithm, we have that $\left\{L B_{k}\right\}$ is a nondecreasing sequence and it has an upper bound $\min _{y \in D} f(y)$, which ensures the existence of the limit $L B:=\lim _{k \rightarrow \infty} L B_{k} \leq \min _{y \in D} f(y)$. Since the sequence $\left\{y^{k}\right\}$ is included in a compact set $Y^{0}$, there must exist a convergent subsequence $\left\{y^{s}\right\} \subseteq\left\{y^{k}\right\}$ and assume that $\lim _{s \rightarrow \infty} y^{s}=y^{*}$. Then by the branching process and deleting method, there must exist a decreasing subsequence $\left\{Y^{r}\right\} \subset Y^{s}$, where $\left(Y^{s}, Z^{s}\right) \in \Lambda_{s}$ with $y^{r} \in Y^{r}$, $L B_{r}=L B\left(Y^{r}\right)=f^{L}\left(y^{r}\right)$ and $\lim _{r \rightarrow \infty} Y^{r}=\left\{y^{*}\right\}$. By the continuity of function $\Psi_{0}(y)$, we have

$$
\begin{aligned}
\lim _{r \rightarrow \infty} L B_{r} & =\lim _{r \rightarrow \infty} f^{L}\left(y^{r}\right) \\
& =\lim _{r \rightarrow \infty} f\left(y^{r}\right) \\
& =f\left(y^{*}\right) .
\end{aligned}
$$

Then, since $Y^{0}$ is a closed set and $\left\{y^{k}\right\} \subset Y^{0}$, obviously, we have that $y^{*} \in Y^{0}$, i.e., $y^{*}$ is a feasible solution of the (NQP). Thus, the proof is completed.

\section{Numerical experiments}

To verify the performance of the proposed algorithm, some numerical experiments are reported and compared with the known methods [19-25]. The algorithm is implemented by Matlab 2016a, and the tests are run in a microcomputer with Intel(R) Xeon(R) processor of $2.4 \mathrm{GHz}, 4 \mathrm{~GB}$ of RAM memory, under the Win10 operational system. We used linprog solver to solve all linear programming problems, and the convergent error is set to $\varepsilon$ in our procedure. For these test examples, the numerical results compared with the current algorithms are demonstrated in Tables 1 and 2, where the following notations have

Table 1 Numerical comparisons for test Examples 1-6

\begin{tabular}{llllrc}
\hline E.g. & Methods & Opt. val. & Opt. sol. & Iter. & Time $(s)$ \\
\hline 1 & ours & 10.00000 & $(2.0000,8.0000)$ & 3 & 0.35725 \\
& {$[25]$} & 10.0000090 & $(1.9999998,7.9999998)$ & 41 & 0.02 \\
& {$[26]$} & 10 & $(2,8)$ & 27 & 10.83 \\
& {$[27]$} & 10 & $(2,8)$ & 53 & 0.3 \\
2 & ours & 3.00000 & $(0.0000,4.0000)$ & 8 & 0.01791 \\
& {$[28]$} & 3.00000 & $(0.0000,4.0000)$ & 25 & 0.750 \\
3 & ours & 0.890185 & $(1.3148,0.1396,0.0,0.4233)$ & 1 & 0.38982 \\
& {$[27]$} & 0.8902 & $(1.3148,0.1396,0.0,0.4233)$ & 1 & 0.1880 \\
& {$[29]$} & 0.890193 & $(1.314792,0.13955,0.0,0.423286)$ & 6 & \\
4 & ours & -16.22662 & $(0.0,3.6403,0.0,2.9029,1.9388,0.0)$ & 5 & 0.59005 \\
5 & ours & -3.00000 & $(3.0000,3.0000)$ & 30 & 4.15970 \\
6 & ours & -1.06250 & $(0.7500,2.0000)$ & 3 & 0.26845 \\
\hline
\end{tabular}


Table 2 Numerical comparisons with Ref. [12, 30] for Example 7

\begin{tabular}{llrrc}
\hline Dimension & Methods & Opt. val. & Iter. & Time (s) \\
\hline$n=5$ & {$[12]$} & -25.0 & 141 & 10.11 \\
& {$[30]$} & -25.0 & 12 & 0.0187 \\
$n=10$ & Ours & -25.0 & 1 & 0.01254 \\
& {$[12]$} & -100.0 & 283 & 21.86 \\
& {$[30]$} & -100.0 & 31 & 0.3342 \\
$n=20$ & ours & -100.0 & 7 & 0.25649 \\
& {$[12]$} & -400.0 & 651 & 47.00 \\
& {$[30]$} & -400.0 & 86 & 5.9396 \\
$n=30$ & ours & -400.0 & 15 & 2.73556 \\
& {$[12]$} & -900.0 & 965 & 106.33 \\
& {$[30]$} & -900.0 & 204 & 44.8577 \\
$n=50$ & ours & -900.0 & 18 & 11.25635 \\
& {$[12]$} & -2500.0 & 1891 & 304.30 \\
$n=80$ & ours & -2500.0 & 21 & 17.35219 \\
$n=100$ & ours & -6400.0 & 37 & 40.45623 \\
$n=150$ & ours & $-10,000.0$ & 51 & 44.35865 \\
& ours & $-22,500.0$ & 66 & 112.99298 \\
\hline
\end{tabular}

been used in column headers: Opt.Val.: optimal value; Opt.Sol.: optimal solution; Iter.: the number of iterations.

Example 1 (Refs. [25-27])

$$
\begin{cases}\min \left(y_{1}+y_{2}\right)\left(y_{1}-y_{2}+7\right) \\ \text { s.t. } & 2 y_{1}+y_{2} \leq 14, \\ & y_{1}+y_{2} \leq 10, \\ & -4 y_{1}+y_{2} \leq 0, \\ & 2 y_{1}+y_{2} \geq 6, \\ & y_{1}+2 y_{2} \geq 6, \\ & y_{1}-y_{2} \leq 3, \\ & y_{1} \leq 5 \\ & y_{1}+y_{2} \geq 0, \\ & y_{1}-y_{2}+7 \geq 0 .\end{cases}
$$

Example 2 (Ref. [28])

$$
\left\{\begin{array}{l}
\min y_{1}+\left(2 y_{1}-3 y_{2}+13\right)\left(y_{1}+y_{2}-1\right) \\
\text { s.t. } \quad-y_{1}+2 y_{2} \leq 8 \\
\quad-y_{2} \leq-3 \\
y_{1}+2 y_{2} \leq 12 \\
y_{1}-2 y_{2} \leq-5 \\
y_{1}, y_{2} \geq 0
\end{array}\right.
$$


Example 3 (Refs. [27, 29])

$$
\left\{\begin{array}{cc}
\min \left(0.813396 y_{1}+0.67440 y_{2}+0.305038 y_{3}+0.129742 y_{4}+0.217796\right) \\
\times & \left(0.224508 y_{1}+0.063458 y_{2}+0.932230 y_{3}+0.528736 y_{4}+0.091947\right) \\
\text { s.t. } \quad & 0.488509 y_{1}+0.063565 y_{2}+0.945686 y_{3}+0.210704 y_{4} \leq 3.562809 \\
& -0.324014 y_{1}-0.501754 y_{2}-0.719204 y_{3}+0.099562 y_{4} \leq-0.052215 \\
& 0.445225 y_{1}-0.346896 y_{2}+0.637939 y_{3}-0.257623 y_{4} \leq 0.427920 \\
& -0.202821 y_{1}+0.647361 y_{2}+0.920135 y_{3}-0.983091 y_{4} \leq 0.840950 \\
& -0.886420 y_{1}-0.802444 y_{2}-0.305441 y_{3}-0.180123 y_{4} \leq-1.353686 \\
& -0.515399 y_{1}-0.424820 y_{2}+0.897498 y_{3}+0.187268 y_{4} \leq 2.137251 \\
& -0.591515 y_{1}+0.060581 y_{2}-0.427365 y_{3}+0.579388 y_{4} \leq-0.290987 \\
& 0.423524 y_{1}+0.940496 y_{2}-0.437944 y_{3}-0.742941 y_{4} \leq 0.373620 \\
& y_{1} \geq 0, y_{2} \geq 0, y_{3} \geq 0, y_{4} \geq 0
\end{array}\right.
$$

\section{Example 4}

$$
\begin{cases}\min & 6.5 y_{1}-0.5 y_{1}^{2}-y_{2}-2 y_{3}-3 y_{4}-2 y_{5}-y_{6} \\ \text { s.t. } & y_{1}+2 y_{2}+8 y_{3}+y_{4}+3 y_{5}+5 y_{6} \leq 16, \\ & -8 y_{1}-4 y_{2}-2 y_{3}+2 y_{4}+4 y_{5}-y_{6} \leq-1, \\ & 2 y_{1}+0.5 y_{2}+0.2 y_{3}-3 y_{4}-y_{5}-4 y_{6} \leq 24 \\ & 0.2 y_{1}+2 y_{2}+0.1 y_{3}-4 y_{4}+2 y_{5}+2 y_{6} \leq 12, \\ & -0.1 y_{1}-0.5 y_{2}+2 y_{3}+5 y_{4}-5 y_{5}+3 y_{6} \leq 3, \\ & 0 \leq y_{i} \leq 10, i=1,2, \ldots, 6\end{cases}
$$

Example 5

$$
\begin{cases}\min & 2 y_{1}+3 y_{2}-2 y_{1}^{2}-2 y_{2}^{2}+2 y_{1} y_{2} \\ \text { s.t. } & -y_{1}+y_{2} \leq 1 \\ & y_{1}-y_{2} \leq 1 \\ & -y_{1}+2 y_{2} \leq 3 \\ & 2 y_{1}-y_{2} \leq 3 \\ & 0 \leq y_{1} \leq 15,0 \leq y_{2} \leq 15 .\end{cases}
$$

\section{Example 6}

$$
\left\{\begin{array}{l}
\min y^{T} Q y+c^{T} y \\
\text { s.t. } \quad A y \leq b, \\
\quad y \in Y^{0}=\left\{0 \leq y_{1} \leq 2,0 \leq y_{2} \leq 2\right\},
\end{array}\right.
$$

where $c=(2,4)^{T}, b=(1,2,4,3,1)^{T}, Q=\left(\begin{array}{cc}-1 & 2 \\ 2 & -4\end{array}\right), A=\left(\begin{array}{cc}-4 & 2 \\ 0 & 1 \\ 1 & 1 \\ 1 & 0 \\ 1 & -4\end{array}\right)$. 
Example 7 (Ref. [12])

$$
\left\{\begin{array}{l}
\min -\sum_{i=1}^{n} y_{i}^{2} \\
\text { s.t. } \quad \sum_{i=1}^{j} y_{i} \leq j, j=1,2, \ldots, n, \\
\quad y_{i} \geq 0, i=1,2, \ldots, n .
\end{array}\right.
$$

Table 2 lists the numerical results of our algorithm, Gao's algorithm [12], and Jiao's algorithm [30] on Example 7. By comparing the numerical results in Table 2, we can conclude that our algorithm applied to Example 7 is superior to Gao's algorithm [12] and Jiao's algorithm [30] in terms of number of iterations and time.

Some randomly generated test examples with a large scale number of variables and constraints are used to validate the robustness of the proposed algorithm. These randomly generated examples and their computational results are given as follows.

\section{Example 8}

$$
\begin{cases}\min y^{T} Q y+c^{T} y \\ \text { s.t. } & A y \leq b, \\ & y \in Y^{0}=\left\{-10 \leq y_{i} \leq 10, i=1,2, \ldots, n\right\},\end{cases}
$$

where $Q_{n \times n}$ is a real symmetric matrix, all the real elements of $Q_{n \times n}, A_{m \times n}$, and $c_{n \times 1}$ are randomly generated in Interval $[-2,2]$, all the real elements of $b_{m \times 1}$ are randomly generated in Interval $[1,10]$. For Example 8, we solved 10 different random instances for each size and presented statistics of the results. The computational results are summarized in Table 3, where the following notations have been used in column headers: Ave.Iter.: the average number of iterations; Ave.Time (s): the average CPU execution time for the algorithm in seconds; $m$ : the number of constraints; $n$ : the number of variables.

From Table 3 and Fig. 1, it can be seen that, when $m$ and $n$ are below 50, the algorithm can find the global optimal solution in a short time and with lower iteration number. As the problem size becomes larger, the average number of iterations and the average CPU time of our algorithm are also increased, but they are not very sensitive to the problem size.

Table 3 Numerical results for Example 8

\begin{tabular}{rrrr}
\hline$n$ & $m$ & Ave.lter. & Ave.Time $(s)$ \\
\hline 5 & 5 & 1.2 & 0.5089 \\
10 & 10 & 1.2 & 1.1423 \\
15 & 10 & 1.3 & 4.7854 \\
30 & 30 & 1.3 & 8.9569 \\
40 & 40 & 1.2 & 8.4528 \\
50 & 50 & 2.5 & 21.3985 \\
80 & 80 & 2.4 & 33.3828 \\
100 & 50 & 3.6 & 29.3468 \\
100 & 100 & 5.4 & 64.5159 \\
200 & 20 & 3.2 & 26.9366 \\
200 & 50 & 3.2 & 42.3695 \\
300 & 50 & 3.5 & 89.3652 \\
300 & 100 & 4.8 & 436.2315 \\
\hline
\end{tabular}




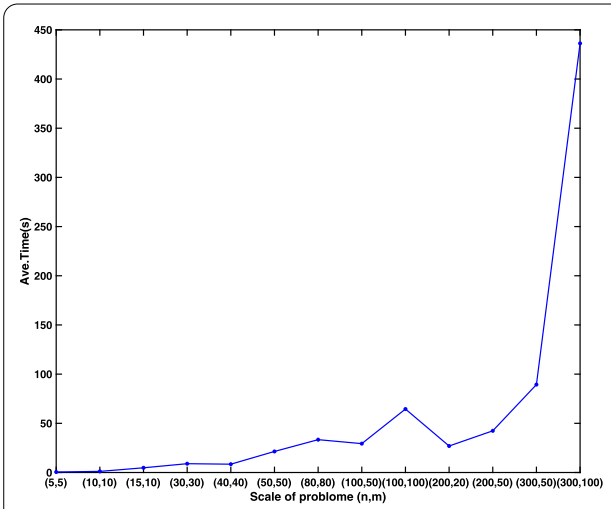

(a)

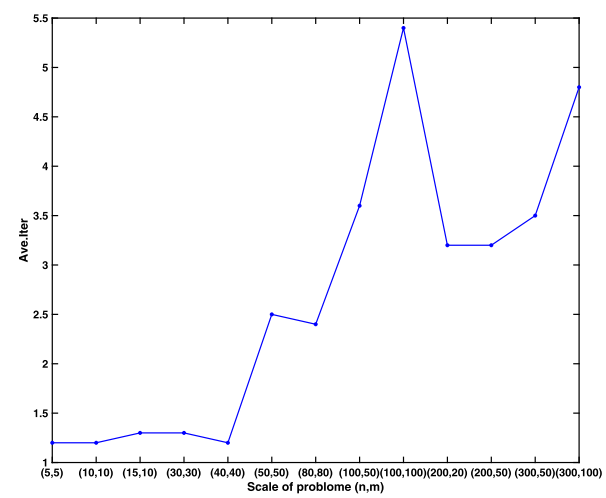

(b)

Figure 1 The variation tendency of performance index with scale of Example 8

From the experimental results in Table 3, we can see that the proposed algorithm with the given convergent error can be used to globally solve the (NQP) with a large scale number of constraints and variables. The results in Tables 1-3 show that our algorithm is both feasible and efficient.

\section{Concluding remarks}

In this paper, we propose a new branch-and-bound algorithm for globally solving the nonconvex quadratic programming problem. In this algorithm, we present a new linear relaxation method, which can be used to derive the linear programs relaxation problem of the investigated problem (NPQ). To accelerate the computational speed of the proposed branch-and-bound algorithm, an interval deleting rule is used to reduce the investigated regions. By subsequently partitioning the initial region and solving a sequence of linear programs relaxation problems, the proposed algorithm is convergent to the global optima of the initial problem (NPQ). Finally, compared with some existent algorithms, numerical results show higher computational efficiency of the proposed algorithm.

\section{Acknowledgements}

The authors are grateful to the responsible editor and the anonymous referees for their valuable comments and suggestions, which have greatly improved the earlier version of this paper.

\section{Funding}

This work is supported by the National Natural Science Foundation of China (No. 61373174, No. 11501168, No. 11602184); the Science and Technology Key Project of Education Department of Henan Province (No. 16A110030).

Competing interests

The authors declare that they have no competing interests.

Authors' contributions

All authors contributed equally to the manuscript, and they read and approved the final manuscript.

\section{Publisher's Note}

Springer Nature remains neutral with regard to jurisdictional claims in published maps and institutional affiliations.

Received: 15 April 2018 Accepted: 4 July 2018 Published online: 16 July 2018

\section{References}

1. Khammash, M.H.: Synthesis of globally optimal controllers for robust performance to unstructured uncertainty. IEEE Trans. Autom. Control 41(2), 189-198(1996) 
2. Kedem, G., Watanabe, H.: Graph-optimization techniques for IC layout and compaction. IEEE Trans. Comput.-Aided Des. Integr. Circuits Syst. 3(1), 12-20 (1984)

3. Lodwick, W.A.: Preprocessing nonlinear functional constraints with applications to the pooling problem. INFORMS J. Comput. 4, 119-131 (1992)

4. Floudas, C.A., Visweswaran, V.: Primal-relaxed dual global optimization approach. J. Optim. Theory Appl. 78(2), 187-225 (1993)

5. Li, Y., Kang, L., Garis, H.D., Kang, Z., Liu, P.: A robust algorithm for solving nonlinear programming problems. Int. J. Comput. Math. 79(5), 523-536 (2002)

6. Shen, C., Xue, W., Pu, D.: A globally convergent trust region multidimensional filter SQP algorithm for nonlinear programming. Int. J. Comput. Math. 86(12), 2201-2217 (2009)

7. Wang, H., Liu, F., Gu, C., Pu, D.: An infeasible active-set QP-free algorithm for general nonlinear programming. Int J. Comput. Math. 94(5), 884-901 (2016)

8. Vandenbussche, D., Nemhauser, G.L.: A branch-and-cut algorithm for nonconvex quadratic programs with box constraints. Math. Program. 102(3), 559-575 (2005)

9. Burer, S., Vandenbussche, D.: Globally solving box-constrained nonconvex quadratic programs with semidefinite-based finite branch-and-bound. Comput. Optim. Appl. 43(2), 181-195 (2009)

10. Cambini, R., Sodini, C.: Decomposition methods for solving nonconvex quadratic programs via branch and bound. J. Glob. Optim. 33(3), 313-336 (2005)

11. Li, H.M., Zhang, K.C.: A decomposition algorithm for solving large-scale quadratic programming problems. Appl. Math. Comput. 173(1), 394-403 (2006)

12. Gao, Y., Xue, H., Shen, P.: A new rectangle branch-and-reduce approach for solving nonconvex quadratic programming problems. Appl. Math. Comput. 168(2), 1409-1418 (2005)

13. Burer, S., Vandenbussche, D.: A finite branch-and-bound algorithm for nonconvex quadratic programming via semidefinite relaxations. Math. Program. 113(2), 259-282 (2008)

14. Al-Khayyal, F.A., Larsen, C., Voorhis, T.V.: A relaxation method for nonconvex quadratically constrained quadratic programs. J. Glob. Optim. 6(3), 215-230 (1995)

15. Linderoth, J.: A simplicial branch-and-bound algorithm for solving quadratically constrained quadratic programs. Math. Program. 103(2), 251-282 (2005)

16. Raber, U.: A simplicial branch-and-bound method for solving nonconvex all-quadratic programs. J. Glob. Optim. 13(4), 417-432 (1998)

17. Sherali, H.D., Adams, W.P.: A Reformulation-Linearization Technique for Solving Discrete and Continuous Nonconvex Problems, vol. 31. Springer, Berlin (1999)

18. Audet, C., Hansen, P., Jaumard, B., Savard, G.: A branch and cut algorithm for nonconvex quadratically constrained quadratic programming. Math. Program. 87(1), 131-152 (2000)

19. Lu, C., Deng, Z., Jin, Q.: An eigenvalue decomposition based branch-and-bound algorithm for nonconvex quadratic programming problems with convex quadratic constraints. J. Glob. Optim. 67(3), 475-493 (2017)

20. Gao, Y., Shang, Y., Zhang, L.: A branch and reduce approach for solving nonconvex quadratic programming problems with quadratic constraints. Oper. Res. Trans. 9(2), 9-20 (2005)

21. Qu, S.J., Zhang, K.C., Ji, Y.: A global optimization algorithm using parametric linearization relaxation. Appl. Math. Comput. 186(1), 763-771 (2007)

22. Shen, P., Duan, Y., Ma, Y.: A robust solution approach for nonconvex quadratic programs with additional multiplicative constraints. Appl. Math. Comput. 201(1-2), 514-526 (2008)

23. Shen, P., Liu, L.: A global optimization approach for quadratic programs with nonconvex quadratic constraints. Chin. J. Eng. Math. 25(5), 923-926 (2008)

24. Shen, P., Wang, C.: Linear decomposition approach for a class of nonconvex programming problems. J. Inequal. Appl. 2017(1), $74(2017)$

25. Chen, Y., Jiao, H.: A nonisolated optimal solution of general linear multiplicative programming problems. Comput. Oper. Res. 36(9), 2573-2579 (2009)

26. Gao, Y., Wu, G., Ma, W.: A new global optimization approach for convex multiplicative programming. Appl. Math Comput. 216(4), 1206-1218 (2010)

27. Wang, C.F., Liu, S.Y., Shen, P.P.: Global minimization of a generalized linear multiplicative programming. Appl. Math Model. 36(6), 2446-2451 (2012)

28. Jiao, H.W., Liu, S.Y., Zhao, Y.F.: Effective algorithm for solving the generalized linear multiplicative problem with generalized polynomial constraints. Appl. Math. Model. 39(23-24), 7568-7582 (2015)

29. Thoai, N.V:: A global optimization approach for solving the convex multiplicative programming problem. J. Glob. Optim. 1(4), 341-357 (1991)

30. Jiao, H., Liu, S., Lu, N.: A parametric linear relaxation algorithm for globally solving nonconvex quadratic programming. Appl. Math. Comput. 250, 973-985 (2015) 